\title{
Retinal artery occlusion after herpes zoster mandibularis in a patient with systemic lupus erythematosus
}

\author{
Gae-Eil Jang and Hyun-Sook Kim
}

Division of Rheumatology, Department of Internal Medicine, Soonchunhyang University Seoul Hospital, Seoul, Korea
Received: October 14, 2018

Revised : November 8, 2018

Accepted: November 8, 2018

\section{Correspondence to}

Hyun-Sook Kim, M.D.

Tel: +82-2-710-3214

Fax: +82-2-709-9554

E-mail: healthyra@schmc.ac.kr
While latent varicella zoster virus (VZV) infections are common, those limited to the facial nerve present as a rare form of VZV reactivation. Herpes zoster mandibularis is characterized as any involvement of the three branches of the trigeminal nerve manifesting as unilateral facial or oral pain. VZV-related vasculopathies can present as ischemic or hemorrhagic stroke, giant cell arteritis, cranial neuropathies, venous sinus thrombosis, spinal cord infarction, and peripheral artery thrombosis, all of which occur after the initial infection. Adults with herpes zoster reactivation exhibit a $31 \%$ increase in stroke frequency in the following year, with reactivations localizing to the ophthalmic area of the trigeminal nerve increasing stroke risk as high as 4.5 -fold. Treatment of VZV vasculopathy may require prolonged intravenous acyclovir.

A 28-year-old female presented with vesicles, pustules along the right lower jaw, and a plaque covering the right half of her tongue (Fig. 1). She had endstage renal disease caused by systemic lupus erythematous and had undergone a right mastectomy due to breast 


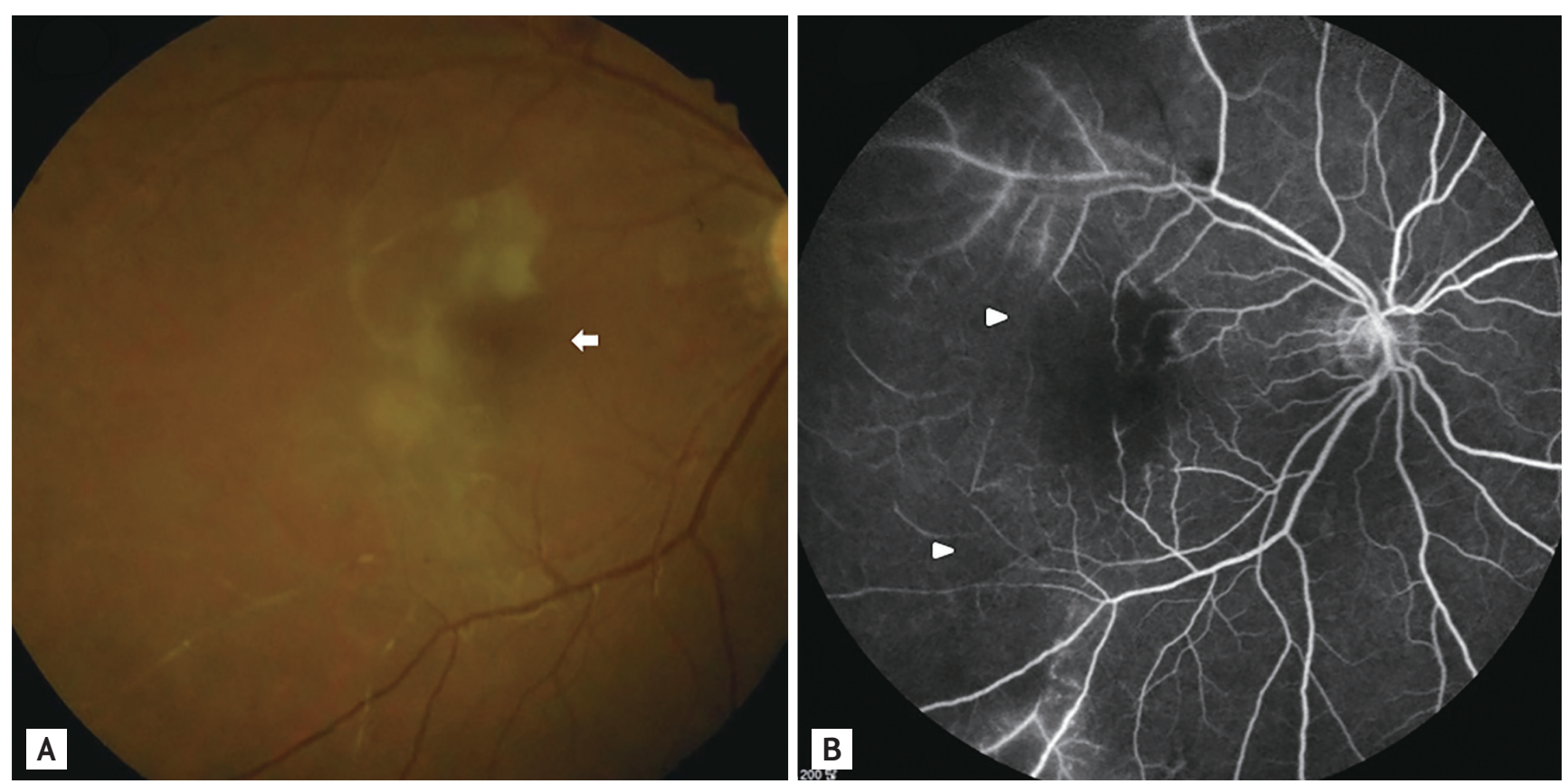

Figure 2. (A) Fundus photography reveals a cherry-red spot and swelling of the retina due to retinal artery occlusion (arrow). (B) Fundus fluorescent angiography reveals decreased perfusion in the peripheral retinal artery due to retinal artery blockage (arrowheads).

cancer in the previous year. She had no antiphospholipid antibodies. Examinations revealed right marginal mandibular palsy. After 2 weeks, right side facial swelling, headache, and visual disturbances had developed. Fundus photography and fluorescent angiography revealed multiple retinal arterial occlusions and swelling of the right eye (Fig. 2). At the time of subsequent follow-up 2 weeks later, neuralgia had subsided and visual disturbances had improved with acyclovir and corticosteroid treatment.

In cases of concomitant herpes zoster mandibularis and VZV vasculopathy, clinical symptoms can present as a wide range of symptoms. Proper diagnosis in such cases relies on a thorough physical examination in combination with a detailed medical history. In cases where a zoster rash is suspected, early initiation of antiviral therapy is very important, as the timing of therapy remains one of the most important factors determining patient outcomes.

We contact her by phone because this study was done after ending the treatment, and verbal consent was obtained. This study was approved by the Institutional Review Board of Soonchunhyang University Seoul Hospital (2018-10-023).

\section{Conflict of interest}

No potential conflict of interest relevant to this article was reported.

\section{Acknowledgments}

This study is supported by fund of the Soonchunhyang University. 